\title{
Radiology-Pathology Correlation of Posterior Fossa Epidermoid Cyst - A Case Report and Short
}

\section{Review of Literature.}

\section{Chithra Ram 1, * MD, Manasaa Kannan², Erik Seroogy ${ }^{3}$, MPH, Terri Mason ${ }^{4}$, BS, MD, Richard Sherry ${ }^{5}$, MD.}

${ }^{1}$ Assistant Professor, Department of Radiology, University of Louisville, Kentucky, USA.

${ }^{2}$ Medical Student, University of Louisville, Kentucky, USA. ${ }^{3}$ Medical Student, University of Louisville, Kentucky, USA. ${ }^{4}$ Department of Pathology, University of Louisville, Kentucky, USA.

${ }^{5}$ Neuroradiologist, RG Sherry MD PLLC, Watertown, New York, USA.

"Corresponding Author: Chithra Ram, Assistant Professor, Department of Radiology, University of Louisville, Kentucky, USA.

Received date: 25 February 2020; Accepted date: 15 March 2021; Published date: 19 March 2021

Citation: Ram C, Kannan M, Seroogy E, Mason T, Sherry R. Radiology-Pathology Correlation of Posterior Fossa Epidermoid Cyst - A Case Report and Short Review of Literature. J Med Case Rep Case Series 2(1): https://doi.org/10.38207/jmcrcs20210013

Copyright: @ 2021 Chithra Ram. This is an open-access article distributed under the terms of the Creative Commons Attribution License, which permits unrestricted use, distribution, and reproduction in any medium, provided the original author and source are credited.

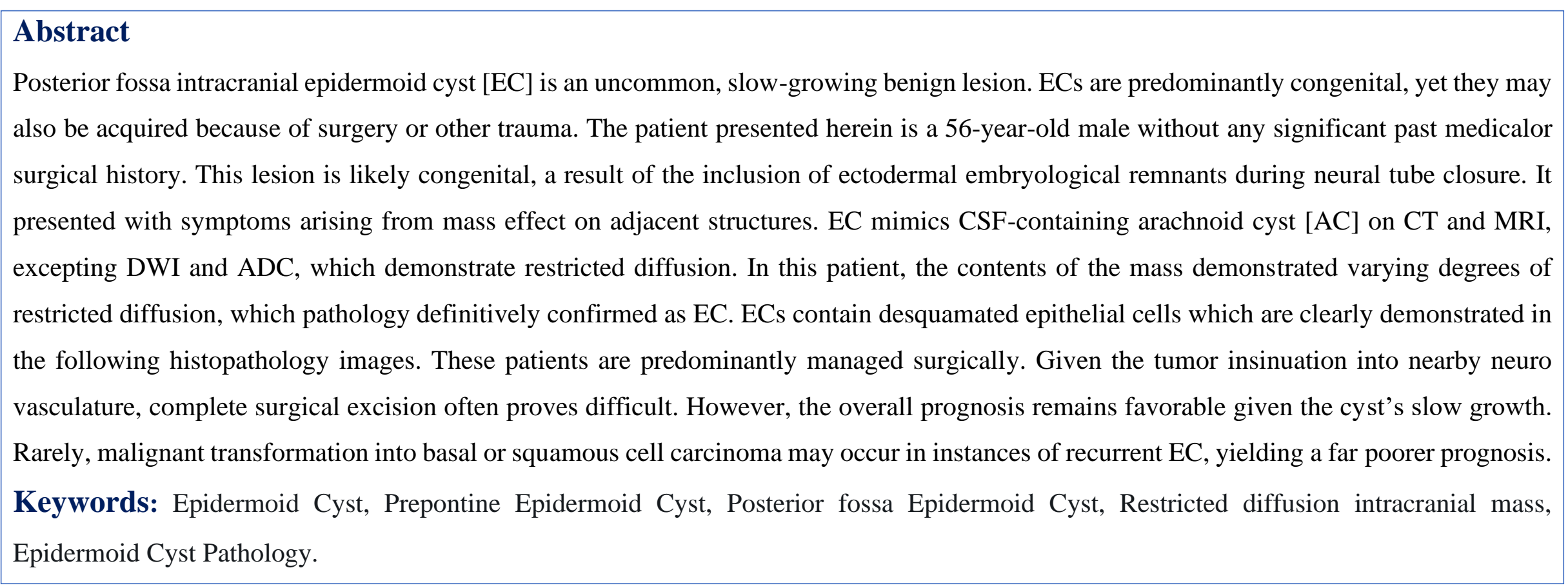

\section{Introduction}

Posterior fossa epidermoid cyst [EC] may be congenital or acquired. This case report demonstrates the Radiology-Pathology correlation of a case of EC obtained with multiple imaging techniques, including non-enhanced CT, MRI with and without contrast, MRI perfusion studies, and histopathology. Though EC appears like an arachnoid cyst $[\mathrm{AC}]$ in imaging, the presence of restricted diffusion on MRI studies differentiates it from AC. ECs contain desquamated keratin and crystallized cholesterol, solely epidermal in nature. In contrast, dermoid cysts [DC] contain both dermal and epidermal elements. Skin appendage components such as hair and sebaceous glands are present alongside the epidermis in DC. Teratoma represents another related entity, comprising of ectodermal, mesodermal, and endodermal elements. As such, it may contain skin, sweat glands, fat, fluid, hair, and teeth.

\section{Case report}

A 56-year-old male with a three-week history of short-term memory loss presented to the ER with a possible syncopal episode. Right cranial nerve VI palsy was present on neurological exam. Brain imaging revealed an extensive prepontine cistern mass complicated by obstructive hydrocephalus. The patient underwent left frontal ventriculoperitoneal shunt placement, with Certas valve set to performance level 3, followed by surgery via right temporal craniotomy for resection of the medial temporal and retro-sigmoid components of the mass, as well as far lateral craniotomy for the posterior fossa component. The entirety of the mass could not be fully removed given its location and infiltration, so the patient is now followed at regular intervals to monitor for interval growth or degeneration of his remaining EC.

\section{Methods}

CT head without contrast was performed in a 128 slice Somatom Edge Siemens scanner [Siemens Medical Systems, USA]. Standard 1 $\mathrm{mm}$ axial CT soft tissue algorithm images of the brain were obtained from the skull base to the vertex, followed by reconstruction coronal images along with axial bone window images. MRI of the brain was performed in a $3 \mathrm{~T}$ Siemens Spectra scanner [Siemens Medical Systems, Malvern, Pennsylvania, USA] using a standard head coil. 


\section{Imaging findings:}

Non-enhanced CT of the brain [Figure 1] shows a large hypodense, irregular, insinuating fluid density mass in the prepontine cistern. It causes mass effect on the pons, bilateral cerebellar hemispheres and right medial temporal lobe. It has associated hydrocephalus. MRI images of the brain [Figure 2, 3] demonstrate a large irregular, insinuating hypointense $\mathrm{T} 1$, hyperintense $\mathrm{T} 2$, hyperintense GRE mass in the prepontine cistern. Though it can follow the cerebrospinal fluid $[\mathrm{CSF}]$ signal in these sequences, it has restricted diffusion which differentiates it from Arachnoid Cyst. In the FLAIR sequence, EC Standard 4 mm thick Axial T1, axial T2, Fat Suppressed Axial T2 FLAIR, axial DWI, and ADC brain pulse sequences were acquired without contrast. Following administration of $20 \mathrm{ml}$ of Multihance, axial and coronal post-contrast sequences were obtained. MRI perfusion of the brain was performed on a 3T Siemens Spectrascanner [Siemens Medical Systems, Malvern, Pennsylvania, USA] using a standard head coil, following administration of $20 \mathrm{ml}$ of Multihance. usually has dirty or low signal, but not as low as CSF. The mass causes mass effect on the nearby pons, bilateral cerebellar hemispheres and right medial temporal lobe. Post contrast MRI images of the brain [Figure 4] do not demonstarte enhancement of posterior fossa mass. MRI Brain Perfusion image [Figure 5] demonstrates decreased perfusion within the large irregular, insinuating mass in the prepontine cistern and extending laterally. The basilar artery has been displaced posteriorly by the mass.

Imaging figures: posterior fossa epidermoid cyst

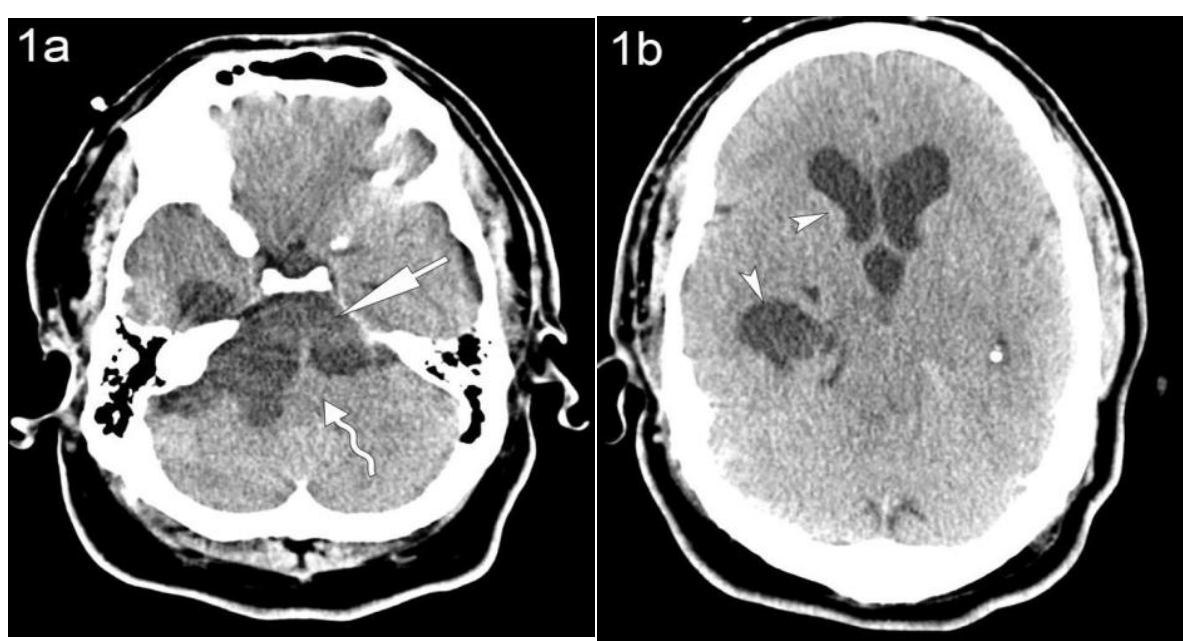

Figure:1. 128 slice Siemens CT scanner Non-enhanced CT head images [1a, 1b].

Axial $(a, b)$ non contrast CT head images demonstrate a large hypodense irregular, insinuating mass in the prepontine cistern (arrow in a), whichcauses mass effect on the pons (squiggly arrow in a) and bilateral cerebellar hemispheres. There is associated hydrocephalus (arrowheads in b) with dilated lateral and third ventricles.
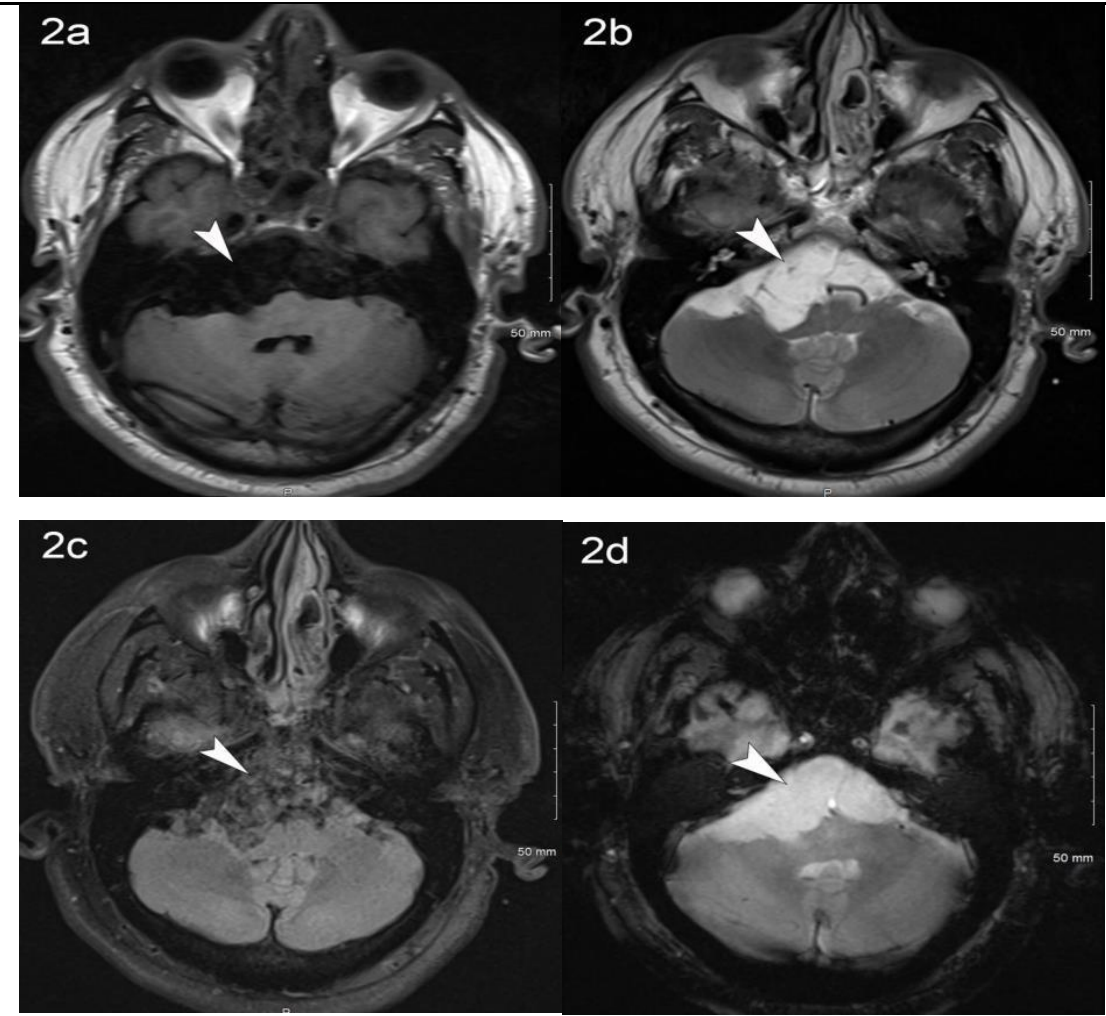

Figure:2 3T Siemens Spectra scanner MRI Brain images without contrast [2a-2d].

Axial T1W (a), axial T2W (b), axial T2 FLAIR (c), axial GRE (d) non contrast MRI brain images demonstrate a large irregular, insinuating hypointense $\mathrm{T} 1$, hyperintense $\mathrm{T} 2$, hypointense/dirty signal T2 FLAIR, hyperintense GRE, mass in the prepontine cistern (arrowhead in a-d), causing mass effect on the nearby pons and cerebellar hemispheres. 


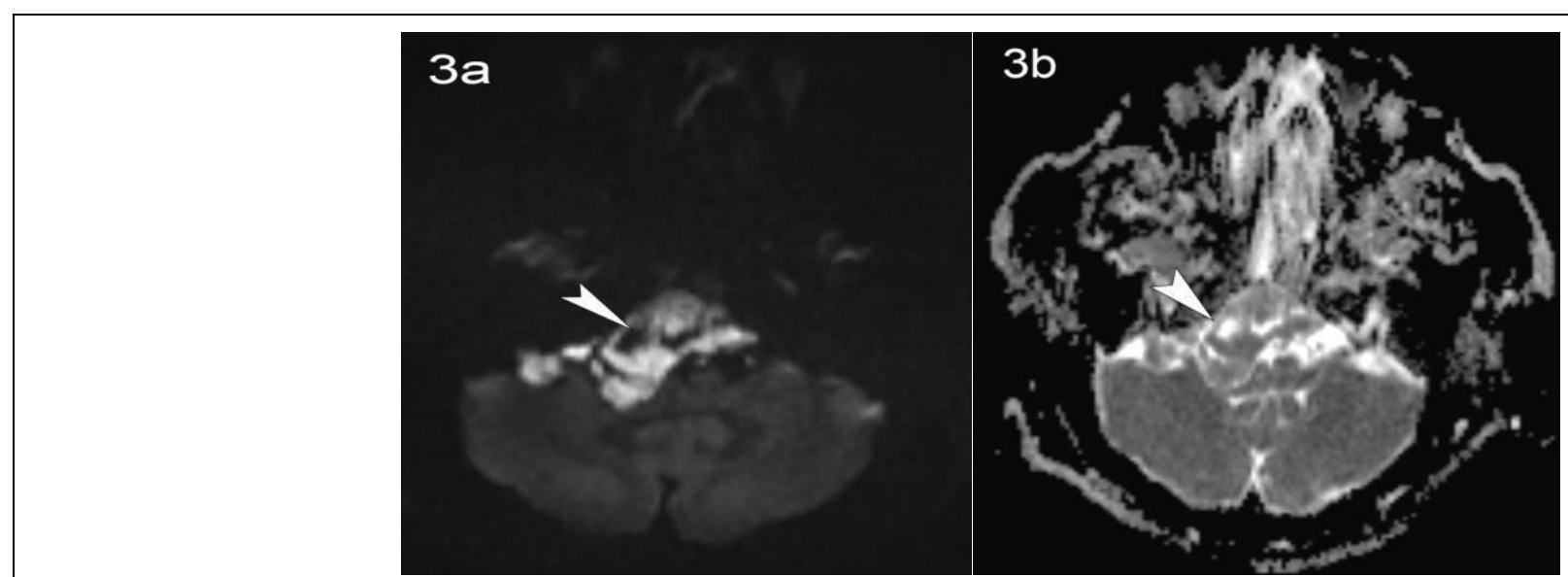

Figure:3. 3T Siemens Spectra scanner MRI Brain non-contrast images [3a, 3b].

Axial DWI (a) and axial ADC (b) non contrast MRI brain images demonstrate a large irregular, insinuating mass in the prepontine cistern (arrowhead in a, b), causing mass effect on the nearby pons and cerebellar hemispheres. It has mixed increased and decreased DWI and ADC signal with varying restricted diffusion within the mass.

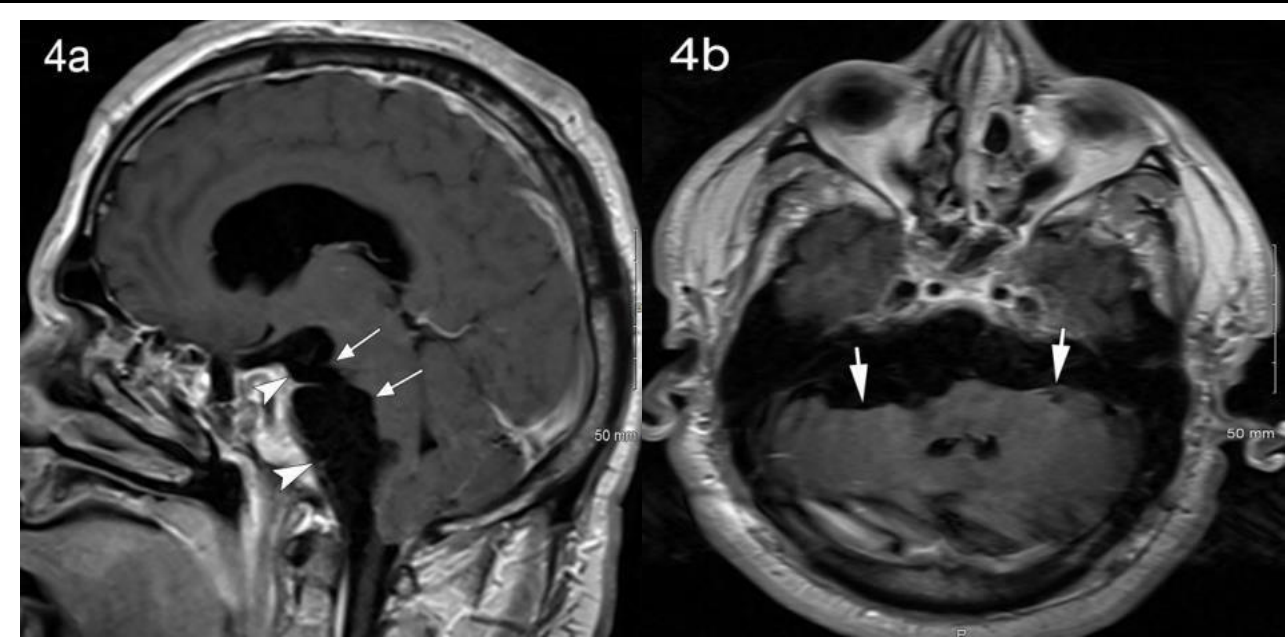

Figure:4 3T Siemens Spectra scanner MRI Brain Post-contrast images [4a, 4b].

Sagittal T1W (a) and axial T1W (b) post-contrast MRI brain images demonstrate a large non-enhancing irregular, insinuating mass in the

pre-pontine cistern (arrowheads in a), b), which causes mass effect on the nearby pons (arrows in a) and cerebellar hemispheres (arrows in b).

Figure:5 3T Siemens Spectra scanner MRI Brain Post-contrast Perfusion image [5].
Axial MRI brain perfusion image post 20cc of IV Multihance contrast demonstrates a large irregular, insinuating mass
in the prepontine cistern with decreased perfusion (arrowhead). Note widening of the prepontine cistern with posterior
displacement of the basilar artery (arrow).

Pathology figures: posterior fossa epidermoid cyst 


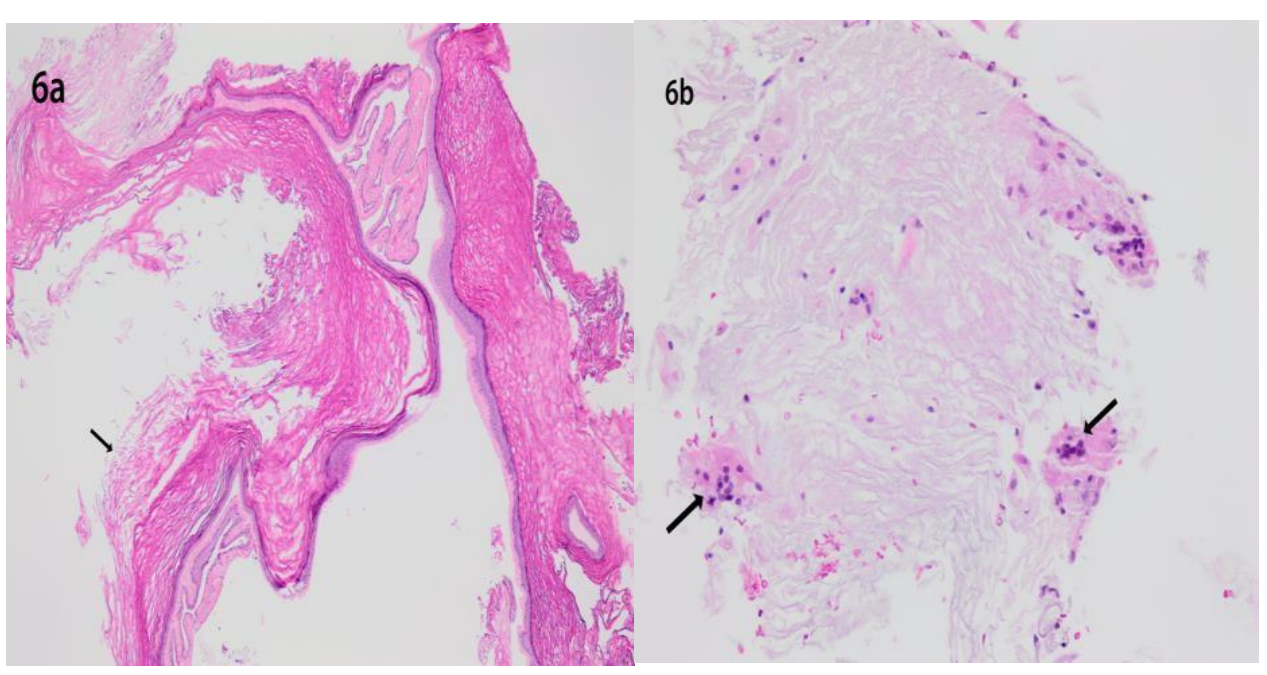

Figure:6. Intraoperative specimen was submitted for frozen section evaluation followed by formalin fixation for routine histopathologyevaluation.

Figure:6a. Low Power H\&E: cyst lining made of stratified squamous epithelium (black arrow) with a prominent granular layer and a central cystic component composed of lamellated keratin flakes.

Figure:6b. High Power H\&E: the cystic component shows nucleate and anucleate keratinizing squamous cells surrounded by keratin debris. There are also few scattered multinucleated giant cells (black arrow).

\section{Histopathology findings:}

The intraoperative specimen was submitted for frozen section evaluation and then subsequently placed in formalin for regular histopathology evaluation. Grossly, the specimen consisted of pearly, white/tan irregular soft tissue. Histopathology showed the cyst was composed of a wall of stratified squamous epithelium with a granular

\section{Discussion}

Introduction: Epidermoid cyst (EC), or epidermal inclusion cyst, is a congenital or acquired inclusion cyst. They most commonly developon the face, neck, trunk, perineum and intracranially, but can occur anywhere [1]. The mass arises from included epidermal remnants during neural tube closure and disjunction of the surface ectoderm with neural ectoderm during embryogenesis [2]. Intracranial epidermoid cysts are congenital lesions that form $0.2-1.8$ $\%$ of all primary intracranial tumors, $40 \%$ of which arise at the cerebellopontine angle $[3,4]$. The distinction between ECs and Epidemiology:

As ECs are slow-growing, patients may begin experiencing symptoms anytime throughout their second to seventh decades of life, though most commonly between 30 and 40 years of age. Young adult males are relatively more affected [9]. $90 \%$ of intracranial ECs are intradural in location, occurring primarily within the cerebellopontine angle, 4th ventricle, basal and parasellar cisterns, or middle/posterior cranial fossa [2]. $10 \%$ are extradural and involve the intradiploic frontal, parietal, occipital, or sphenoid skull. It can uncommonly

\section{Clinical presentation:}

Clinical symptoms depend on location and degree of mass effect on adjacent neurovascular structures. Headache is the most reported layer, as well as a cystic component filled with lamellated keratin flakes (nucleate and anucleate keratinizing squamous cells). There were several scattered multinucleated giant cells seen within the keratin debris. Findings were consistent with EC.

dermoid cysts [DC] is the presence of sebaceous glands and other elements of the skin epidermis and dermis within the DC [5]. Approximately $1 \%$ of ECs transform into malignant basal or squamous cell carcinoma [6]. Symptomology arises when the EC impinges upon local structures, such as the 4th ventricle, trigeminal nerve, internal carotid artery, or other adjacent neurovasculature [7]. EC may rarely be associated with Currarino syndrome, which presents with a constellation of associated anomalies of anorectum, sacrum, and presacral soft tissue [8].

occur in the cerebral hemispheres and prepontine cistern, as well as rarely occur in the brainstem and within the tela choroidea of the temporal horn, 3rd, or 4th ventricles. They have also been occasionally documented in the cerebellar hemisphere, Sylvian fissure, and suprasellar region [2]. White/dense EC represents an uncommon variant and forms $3 \%$ of all intracranial EC, with similar locations but possessing female predominance of 2.5 to $\mathbf{1}$ [2].

symptom [10]. Patients may experience neuropathy of cranial nerves V, VI, VII, and VIII. Symptoms are either caused by mass effect on 
surrounding neural structures or less commonly, aseptic meningitis secondary to rupture and inflammation caused by leaked cyst contents [2]. Cerebellopontine angle ECs yield more generalized symptoms related to increased intracranial pressure (i.e., headache,

\section{Imaging findings:}

CT - Epidermoid cyst is a lobulated, irregular cauliflower-shaped mass with "fronds". It is hypodense like CSF and does not enhance. It insinuates into adjacent cisterns and encases nearby neurovasculature.

MRI - It has decreased T1 signal, increased T2 signal and does not enhance. In FLAIR, EC usually has dirty or low signal but higher than CSF, as it does not completely null. Restricted diffusion is pathognomonic of EC and helps distinguish it from AC. EC with hemorrhage shows mixed density/intensity in CT/MRI secondary to methemoglobin. The keratin composition of the mass results in both

\section{Histopathology findings:}

On gross examination, EC is a round to oval-shaped, well-defined, smooth mass with a white/pearly outer capsule and containing keratinaceous waxy material [11,15]. On histology, EC is characterized by its orderly stratified squamous epithelium with a distinct granular layer and cystic component containing lamellated keratin flakes, crystalline cholesterol, and additional keratin debris [16]. The acellular keratin flakes are eosinophilic and focally basophilic [17]. Avascular stratified squamous epithelium makes up the cyst's wall [16]. Foreign body giant cell reaction is frequently present in ruptured cysts [18]. Calcification is rarely seen in EC [16]. Dermoid Cysts differ from ECs in that Dermoid Cysts contain skin

\section{Prognosis and treatment:}

The treatment of choice is complete surgical resection, but this may not always be possible due to anatomical complexity and/or mass surrounding neurovascular structures. There has also been some success with conservative treatment via aspiration of cyst contents. However, recurrence is common $(\sim 20 \%)$ if the EC is only partially removed. Post-surgical adjuvant radiotherapy may decrease the likelihood of recurrence [2]. Gamma knife radiosurgery may lessen the clinical burden of symptomatic EC which cannot be resected [4]. Post-surgical complications are dependent on the surgical approach and can include aseptic meningitis and cranial nerve palsy [10]. For

\section{Conclusion}

EC is a slow-growing, benign entity. It tends to insinuate into nearby structures and encase neuro vasculature structures leading to clinical manifestations from mass effect, prompting brain imaging and surgical intervention. This case report demonstrates EC findings in multiple imaging modalities using multiple imaging techniques along with its pathologic correlation. EC may mimic CSF-containing AC on imaging, with the pathognomonic exception of restricted diffusion in DWI and ADC sequences. It is important that DWI studies be included in routine brain imaging sequences to confidently papilledema), seizures, cerebellar ataxia, and/or more localized symptoms (i.e., vertigo, ophthalmoplegia, tinnitus, trigeminal neuralgia) due to impingement of the cranial nerves [10,11]. Altered mental status may rarely be seen in cases of severe mass effect [12].

long relaxation of $\mathrm{T} 1$ and $\mathrm{T} 2$, and no postcontrast MR enhancement [11]. MRI perfusion is decreased in intracranial EC, as it is not a vascular lesion. Dense (CT) or white (MRI) epidermoid is an uncommon variant, which is presumed to be caused by the high protein concentration of the cystic fluid [13]. In CT, it has a higher density than CSF. In MRI, it has heterogeneous CSF signal intensity with high $\mathrm{T} 1$ and low $\mathrm{T} 2$ signals, which may be due to their proteinaceous lipid content, rich in fatty acids but devoid of cholesterol [14].

appendages (hair follicles, sebaceous glands, and sweat glands) [19]. Both are positive for cytokeratin or epithelial membrane antigen (EMA) on immunohistochemical staining [16].

Immunohistochemical staining shows lining cells positive for EMA, negative for GFAP and S-100 protein. Arachnoid Cysts have no epithelial lining and are instead lined by meningothelial cells. Ependymal cysts have an epithelial lining with low cuboidal to columnar cells that are frequently ciliated. Immunohistochemical staining of ependymal cysts shows a lining positive for GFAP and S100 [19].

the uncommon cases of aseptic meningitis, which are typically selflimiting, steroids may be utilized. Additionally, prophylactic postsurgical steroid tapering has been proposed as a prevention measure. If untreated, hydrocephalus may complicate the clinical course, possibly requiring CSF shunting [20]. EC may rarely undergo malignant degeneration into basal or squamous cell carcinoma, which is indicated by accelerated growth and/or post-contrast enhancement [21]. Malignant transformation of EC carries a poor prognosis, but this is quite rare and almost solely limited to cases of recurrent EC $[2,3]$.

differentiate EC from AC. Typically, ECs demonstrate heterogeneous areas of both high and low signal on diffusion-weighted images indicating varying degrees of restricted diffusion internally. EC also has dirty or slightly heterogeneous signal in FLAIR sequence, and not as low as CSF. MRI perfusion confirms the avascular nature of the mass. Surgical resection of EC is the treatment of choice. However, in the presence of neurovasculature encasement, EC may not be amenable for complete resection and hence necessitate continuous monitoring. Overall, EC holds a favourable prognosis, which declines 


\author{
Abbreviations \\ EC - Epidermoid CystAC - Arachnoid cyst DC - Dermoid cyst \\ MRI -Magnetic Resonance ImagingCT - Computerized Tomography \\ CSF - Cerebrospinal fluid
}

\section{Conflict of interest: None}

\section{References}

1. Vaughan VC, Wisell J (2020) Epidermal (epidermoid) type. PathologyOutlines.com Website.

2. Patibandla, M. R., Yerramneni, V. K., Mudumba, V. S., Manisha,N., Addagada, G. C (2016) Brainstem epidermoid cyst: An update. Asian Journal of neurosurgery, 11(3): 194-200.

3. Roh, T. H., Park, Y. S., Park, Y. G., Kim, S. H., Chang, J. H (2017) Intracranial squamous cell carcinoma arising in a cerebellopontine angle epidermoid cyst: A case report and literature review. Medicine, 96(51): e9423.

4. El-Shehaby AMN, Reda WA, Karim KMA, Eldin RMA, Nabeel AM (2017) Gamma knife radiosurgery for cerebellopontine angleepidermoid tumors. Surg Neurol Int. 8: 258.

5. Balasundaram P, Garg A, Prabhakar A, Devarajan L, S, J, Gaikwad SB, et al. (2019) Evolution of epidermoid cyst into dermoid cyst: embryological explanation and radiologicalpathological correlation. Neuroradiol J. 32(2): 92-97.

6. Hoang VT, Trinh CT, Nguyen $\mathrm{CH}$, Chansomphou V, Chansomphou V, et al. (2019) Overview of epidermoid cyst. Eur J Radiol Open. 6: 291-301.

7. Warakulle DR, Anslow P (2003) Differential diagnosis of intracranial lesions with high signal on T1 or low signal on T2weight MRI. Clin Radiol. 58(12): 922-933.

8. Kocaoglu M, Frush DP (2006) Pediatric presacral masses. Radiographics. 26(3): 833-857.

9. de Mendonca JCG, Jardim ECG, Dos Santos CM, Masocatto DC,de Quadros DC, et al. (2017) Epidermoid cyst: clinical and surgical case report. Ann Maxillofac Surg. 7(1): 151-154.

10. deSouza C E, deSouza R, da Costa S, Sperling N, Yoon T. H, et al. (1989). Cerebellopontine angle epidermoid cysts: a report on 30 cases. Journal of neurology, neurosurgery, and psychiatry. 52(8), 986-990.

11. Chen CY, Wong JS, Hsieh SC, Chu JS, Chan WP, et al. (2006)
FLAIR - Fluid attenuated inversion recovery

DWI - Diffusion Weighted Imaging

ADC - Apparent Diffusion Coefficient.

Funding: None

Intracranial epidermoid cyst with hemorrhage: MR imaging findings. Am J Neuroradiol. 27(2): 427-429.

12. Sirin S, Gonul E, Kahraman S, Timurkaynak E (2005) Imaging ofposterior fossa epidermoid tumors. Clin Neurol Neurosurg. 107(6): 461-7.

13. I H Tekkök 1, O Cataltepe, S Sağlam (1991) Dense epidermoid cyst of the cerebellopontine angle. Neuroradiology. 33(3):2557.

14. Horowitz L, Chari MV, James R, Bryan RN (1990) MR of intracranial epidermoid tumors: correlation of in vivo imaging with in vitro 13C spectroscopy. Am J Neuroradiol. 11(2): 299302.

15. Ochi M, Hayashi K, Hayashi T, Morikawa M, Ogino A, et al. (1998) Unusual CT and MR appearance of an epidermoid tumor of the cerebellopontine angle. Am J Neuroradiol. 19(6): 11131115.

16. Rivera-Zengotita M. L, Yachnis A. T (2014). Neuropathology: A Volume in the High Yield Pathology Series. Elsevier.

17. Lester, S. C (2018). Diagnostic Pathology: Intraoperative consultation (2nd ed.). Amsterdam: Elsevier.

18. Gao PY, Osborn AG, Smirniotopoulos JG, Harris CP (1992) Radiologic-pathologic correlation: epidermoid tumor of the cerebellopontine angle. Am J Neuroradiol. 13(3): 865-72.

19. Gattuso P (2014). Differential Diagnosis in Surgical Pathology (3rd ed.). Elsevier.

20. Chowdhury FH, Haque MR, Sarker MH (2013) Intracranial epidermoid tumor; microneurosurgical management: an experience of 23 cases. Asian J Neurosurg. 8(1): 21-28.

21. Link MJ, Cohen PL, Breneman JC, Tew JM (2002) Malignant squamous degeneration of a cerebellopontine angle epidermoid tumor. J Neurosurg. 97(5): 1237-43. 